\title{
Razonamiento cinemático en mecanismos eslabonados a través de ambientes computacionales
}

Chained mechanisms kinematic reasoning

Resumen: Este artículo se desarrolla a partir de los resultados obtenidos en el proyecto de investigación denominado Razonamiento espacial en cinemática de mecanismos, patrocinado por la Universidad Pedagógica Nacional a través del CIUP. Mediante el uso de un hipertexto y un simulador se buscó mejorar las condiciones de percepción e in ferencia del movimiento en el espacio de mecanismos eslabonados. Se evaluaron las condiciones de eficiencia y eficacia para resolver problemas de movimiento de mecanismos, comparando dos grupos de estudiantes del programa de Licenciatura en Diseño Tecnológico. El primer grupo interactuó con el hipertexto y el segundo, con el hipertexto-simulador.

Palabras clave: Razonamiento cinemático, mecanismos eslabonados, razonamiento espacial, hipertexto, simulador, cadenas causales.

\author{
Víctor Ruiz* \\ Nilson Valencia** \\ Artículo recibido: 23-7-2007 y aprobado: 30-4-2008
}

through computational environments

Abstract: This article shows the results obtained from the research project entitled "Spatial Reasoning in kinematics of mechanisms"sponsored by the National Pedagogical University through CIUP. The aim of the study was to improve the conditions of perception and inference of the movement in the space of chained mechanisms, by means of the use of a hypertext and a simulator. The conditions of efficiency and effectiveness were evaluated to solve problems of movement in several mechanisms, by comparing two groups of students who belong to a B.A program in Technological Design. The first group interacted with the hypertext and the second interacted with the hypertext-simulator.

Key words: Spatial reasoning, Mechanisms, Kinematic reasoning, hypertext, simulators, causal events chains.

* Universidad Distrital Francisco José de Caldas, Facultad Tecnológica. veruizr@udistrital.edu.co.

** Universidad Pedagógica Nacional, Departamento de Tecnología.nvalencia@pedagogica.edu.co.

$16 \mathbf{T} \in \boldsymbol{\Delta} \mathrm{N} .{ }^{\circ} 23 \cdot$ Primer semestre de 2008 • pp. 16-30 


\section{Introducción}

Un mecanismo es un arreglo de eslabones que se articulan entre sí por medio de elementos de unión para transmitir y transformar movimiento; por ejemplo, de rotacional continuo a rotacional cíclico, o de rotacional a lineal. Un esquema general de un mecanismo eslabonado se presenta en la figura 1. Este tipo de soluciones tiene una gran cantidad de aplicaciones para resolver problemáticas relacionadas con la transmisión y conversión de movimiento $y$, por tanto, su conocimiento es fundamental para las personas dedicadas a desarrollar este tipo de soluciones.

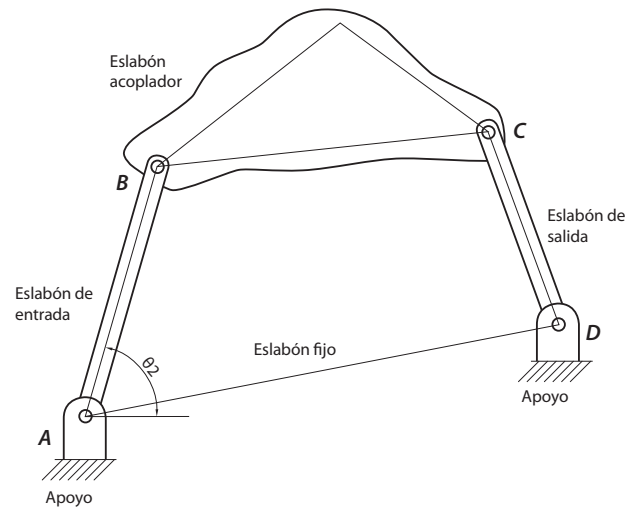

Figura 1. Mecanismo eslabonado de cuatro barras.

En el programa de Licenciatura en Diseño Tecnológico con Énfasis en Sistemas Mecánicos de la Universidad Pedagógica Nacional se detectó por medio de observaciones de campo y pruebas diagnósticas que los estudiantes presentan dificultades conceptuales y metodológicas al analizar mecanismos simples y complejos. Las conclusiones de este análisis sugieren que las deficiencias surgen por el bajo nivel de abstracción espacial en la solución de problemas que involucran el movimiento de los sistemas mecánicos, lo cual redunda en los bajos niveles de comprensión, análisis e interpretación de sistemas tecnológicos al dar solución a necesidades específicas utilizando mecanismos eslabonados. Para contribuir a la solución de esta problemática se diseñó un ambiente computacional compuesto por un hipertexto y un simulador, con el objeto de promover el desarrollo de habilidades en tres aspectos fundamentales:

- reconocer las formas en que los objetos cambian de posición en el espacio,

- determinar las restricciones de movimiento ofrecidas por las uniones que hay entre eslabones, $y$

- verificación del movimiento complejo de un mecanismo como la suma de movimientos simples de sus eslabones para una combinación específica de los mismos.

La interacción con los ambientes computacionales permitió verificar cómo el estudiante es capaz de estructurar la solución a un problema de movimiento de mecanismos teniendo en cuenta los aspectos anotados previamente.

\section{Contexto teórico}

Al ser la cinemática un estudio físico, los modelos utilizados para describir el movimiento son esencialmente matemáticos. Las magnitudes que cualitativamente hacen la descripción física toman cuerpo en las representaciones vectoriales como cantidades que expresan tanto lo cualitativo como lo 
cuantitativo. Los conceptos de posición, velocidad y aceleración determinan la calidad y la cualidad del movimiento. Sin embargo los modelos matemáticos y sus representaciones vectoriales no logran fácilmente generar una representación mental alrededor del movimiento de un mecanismo (Poli, et ál, 2003). Para los estudiantes y profesionales de carreras donde es importante el análisis y la creación de sistemas mecánicos, se requieren habilidades que les permitan entender e inferir el movimiento de un mecanismo sin estar necesariamente planteadas las ecuaciones que gobiernan su comportamiento.

El desarrollo de asistentes computarizados que promuevan y mejoren las habilidades para generar abstracciones del movimiento de los cuerpos en el espacio ha sido abordado por diferentes autores, tal como se enunciarán en adelante, teniendo en cuenta diferentes elementos que intervienen en los procesos cognoscitivos que un individuo lleva a cabo para determinar e inferir el movimiento de un sistema mecánico. La manera en que los individuos pueden percibir y predecir los cambios y las relaciones espaciales que presenta un elemento o conjunto de elementos es fundamental cuando se habla de movimiento, por esto se considera el razonamiento espacial como un eje primordial en la investigación que dio origen a este documento. El manejo de los conceptos de visualización, modelación mental y razonamiento cinemático han sido aportes de diferentes autores en la discusión alrededor de la percepción de movimiento.

\section{Visualización}

Potter y Merwe (2002) desarrollaron estrategias basadas en las teorías piagetianas de la percepción y la imaginería mental, entendida esta última como el conjunto de los procesos mentales que el individuo es capaz de desarrollar para generar representaciones visuales de los objetos que están en el espacio. Tienen en cuenta que estos procesos se agudizan a través de acciones de repetición e imitación del entorno, en especial la representación gráfica. Involucraron a estudiantes de ingeniería en tareas de bocetos, descripciones gráficas y construcción de modelos tridimensionales con el objetivo de aumentar el nivel de percepción, abstracción e imaginería mental.

Murch y Woolf (2002) desarrollaron un tutor que le permite al estudiante crear vistas ortogonales a partir de isométricos, o lo contrario, con diferentes niveles de dificultad. Este tutor viene siendo utilizado en la Universidad de Massachusetts en los cursos básicos de dibujo de ingeniería y busca mejorar los procesos de razonamiento diagramático de los estudiantes a partir de ejercicios que le permitan al estudiante abstraer imágenes y cambiar puntos de vista mentalmente.

Poli et ál.. (2004), plantean que los problemas de visualización y percepción encontrados en una facultad en psicología son los mismos que para una de ingeniería y ciencias de computación. Determinaron que los estudiantes tienen gran dificultad de abordar la visualización, por sí mismos, de las transformaciones espaciales de los obje- 
tos. Buscaron estrategias metodológicas para garantizar el desarrollo de sus habilidades de razonamiento y visualización. Como resultado se elaboraron tutores computarizados adaptables a las necesidades individuales de los estudiantes. La adapatabilidad supone que se requiere comprender mejor el proceso cognitivo que forma el núcleo de la visualización y de las destrezas del razonamiento espacial para la elaboración de herramientas que efectivamente aporten en el desarrollo de estas condiciones mentales.

\section{Modelamiento mental} y razonamiento cinemático

Chandracekara (2000) plantea representaciones perceptuales para resolver problemas visuales de diseño mecánico. Desde aquí plantea el razonamiento cinemático como el proceso de recrear un modelo mental de un sistema formado por elementos que se comportan de acuerdo con las leyes del movimiento mecánico. Esta recreación sugiere que el proceso inferencial implícito en el razonamiento mecánico involucra la simulación mental de la operación del mecanismo.

Hegarty (1992) utilizó mecanismos simples como arreglos de poleas cuerdas y pesos unidos entre sí que le permitieron plantear la pregunta: ¿Cuáles son los procesos involucrados en la inferencia del movimiento de los elementos si el extremo de una cuerda libre es halado? La autora en mención plantea que el proceso de simulación o animación mental de la cinemática de un sistema se hace paso a paso. En esta lógica propone que los modelos mentales de mecanismos específicos se hagan a partir de una serie de pasos que permitan a la persona que está razonando inferir y describir el comportamiento global del mecanismo a partir del comportamiento individual de sus partes y las maneras en que cada parte se relaciona con elementos adyacentes. El conjunto de pasos constituye la animación mental del movimiento del mecanismo.

La animación mental consiste entonces en una serie de procesos cognitivos en los cuales los componentes individuales son animados uno por uno, en lugar de un proceso de animación de todo el sistema. Se genera entonces una cadena causal de eventos donde se hacen sucesivas relaciones entre elementos precedentes y subsiguientes de forma que al sumarse los movimientos individuales es posible describir completamente todo el movimiento de un sistema.

\section{Mecanismos eslabonados}

El movimiento de un mecanismo está definido a partir de sus eslabones y de las uniones que se utilizan para articular los elementos desde la entrada del mecanismo hasta la salida.

\section{Eslabones}

Un eslabón se considera un elemento rígido sin una forma definida específicamente; sin embargo, pueden catalogarse como binarios, ternarios o cuaternarios, según el número de puntos de articulación tengan. Como ejemplo se ven los mecanismos de la figura 2.

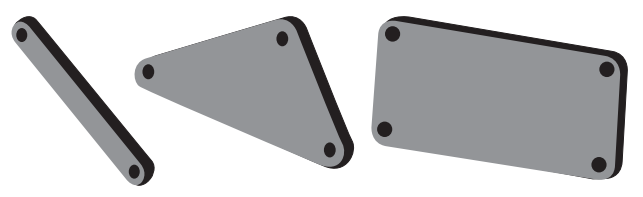

Figura 2. Diferentes tipos de eslabones. Binario terciario y cuaternario 


\section{Uniones}

Las uniones en un mecanismo definen qué tipo de movimiento puede existir entre dos eslabones que se articulan entre sí. De esta manera se tienen, de una forma general, tres tipos de unión, rotacionales, lineales prismáticas y de contacto simple.

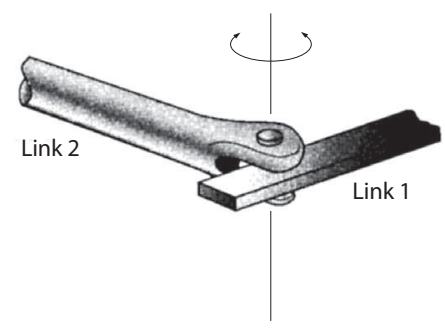

Las uniones de tipo rotacional únicamente permiten movimiento circular alrededor del punto de unión. Las lineales o prismáticas no permiten rotación alguna, únicamente permiten el desplazamiento en la línea recta que define la restricción de la unión (figura 3).

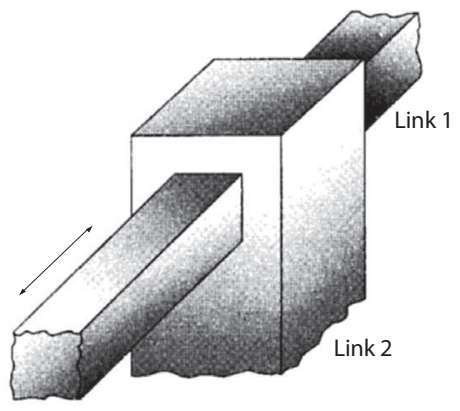

Figura 3. Uniones rotacional y lineal o prismática. Tomado de Joussefii, K. Mechanisms. Berkeley University 2002. Presentación de diapositivas.

En las uniones de contacto simple solo existe un contacto entre las superficies de dos eslabones; sin embargo, no

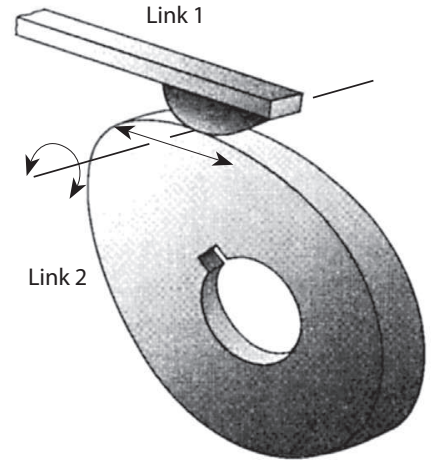

(a) Cam joint hay un elemento adicional que asegure la unión (figura 4).

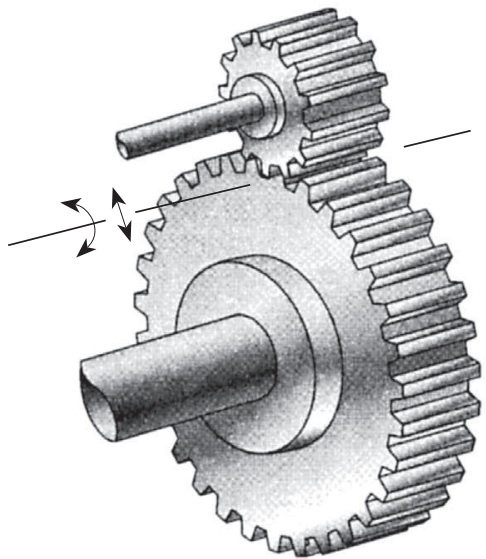

(b) Gear joint

Figura 4. Uniones de contacto simple.Tomado de Joussefii K. Mechanisms. Berkeley University, 2002. Presentación de diapositivas. 


\section{Tipos de movimiento}

De acuerdo con las uniones o restricciones de movimiento y las longitudes de los eslabones se definen las condiciones del movimiento del mecanismo. Los mecanismos planares corresponden a los mecanismos que desarrollan su característica de movimiento en un solo plano, y los tridimensionales, los que definen su movimiento en más de un plano.

\section{Grados de libertad}

Se define como grado de libertad, GDL, los posibles movimientos que en conjunto puede tener un mecanismo. Una definición más rigurosa expresa el número de grados de libertad como una función del número de elementos y sus uniones por medio de la siguiente fórmula:

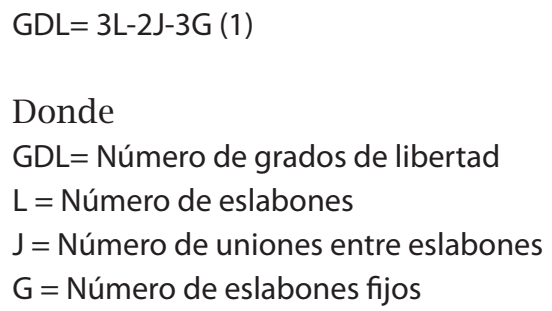

Los mecanismos rotacionales corresponden a los que únicamente tienen uniones rotacionales entre eslabones; la entrada de movimiento será rotacional, generalmente continua y la salida será rotacional continua, o discontinua, oscilante, según las longitudes de los eslabones. Para un mecanismo de cuatro eslabones la rotación continua o discontinua la define la relación de Grashoff (Norton, 2002), que corresponde a

$$
\mathrm{S}+\mathrm{L}<\mathrm{P}+\mathrm{Q}(2)
$$

\section{Donde \\ S es la longitud del eslabón más corto \\ L es la longitud del eslabón más largo, y \\ $\mathrm{P}$ y $\mathrm{Q}$ son las longitudes de los otros dos eslabones}

Cuando la relación de Grashoff se cumple, al menos uno de los dos eslabones de entrada y/o salida rotará continuamente, y se denomina manivela; cuando un eslabón no rota completamente se denomina oscilador. En las figuras 5 y 6 se definen estos casos.

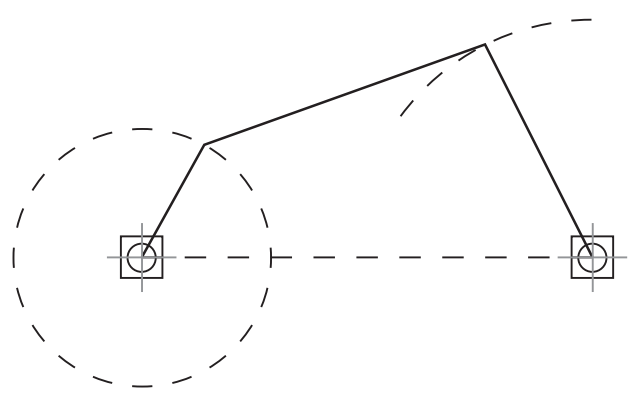

Figura 5. Mecanismo manivela oscilador.

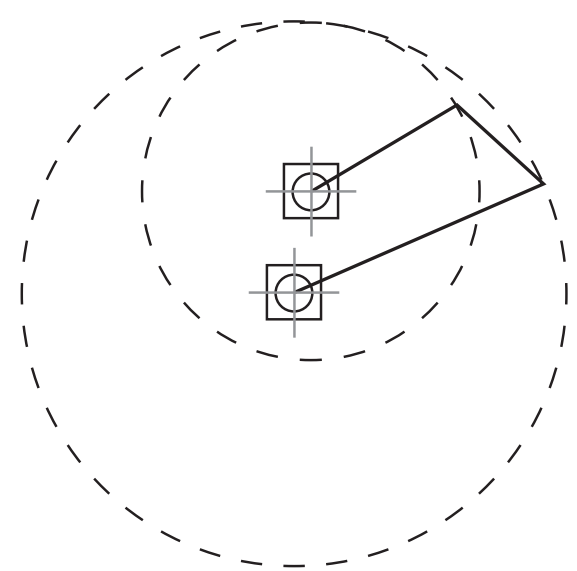

Figura 6. Mecanismo doble manivela. 
Cuando la relación de Grashoff no se cumple se tiene un doble oscilador (figura 7).

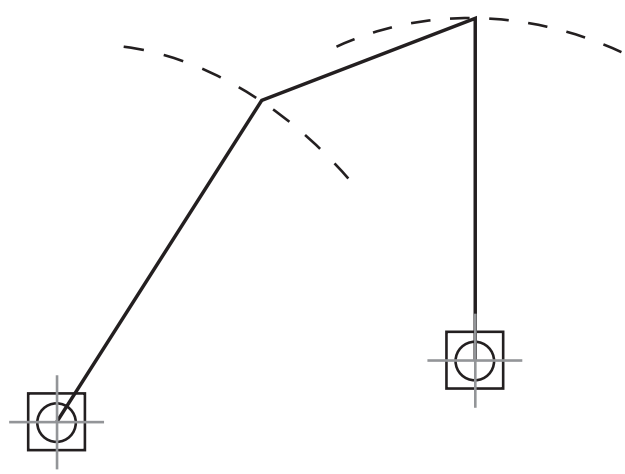

Figura 7. Mecanismo doble oscilador.

\section{Razonamiento espacial y cinemática de mecanismos: una propuesta}

Después de abordar la discusión que se ha llevado alrededor de la aplicación del razonamiento espacial y del análisis no matemático del movimiento de un mecanismo, surge una pregunta: ¿Es posible que un individuo logre describir por completo el movimiento de un mecanismo eslabonado, utilizando modelos o imágenes mentales que le permitan relacionar las interacciones que existen entre elementos para lograr la comprensión del movimiento conjunto de todo el ensamble?

\section{Consideraciones generales}

Hegarty (1999) y Nikam et ál. (2004) han demostrado que es posible que un individuo infiera el movimiento de un arreglo mecánico incluso observando imágenes estáticas de los elementos; sin embargo, en los mecanismos eslabonados no es claro que esta metodología basada en animación mental sea completamente efectiva, esto teniendo en cuenta que el movimiento de los mecanismos no está afectado exclusivamente por los tipos de unión existentes entre elementos y la dirección del movimiento de uno de los eslabones, tal como muestran los estudios referenciados. De acuerdo con el estudio del comportamiento de los mecanismos existe una característica adicional, la geometría del mecanismo, y más específicamente la longitud existente entre los puntos de unión de cada eslabón, tal como lo explica la relación de Grashoff (Norton, 1999). Por tal razón, aparece una tarea adicional que consiste en determinar de forma cualitativa el tamaño de los eslabones, lo cual en ocasiones no es sencillo teniendo en cuenta que en un mecanismo la diferencia entre las longitudes de los eslabones que lo conforman puede ser poco significativa si sólo se le aprecia gráficamente. Esto implicaría una carga adicional en la memoria de trabajo del individuo en el momento de resolver un problema incidiendo negativamente en una solución acertada y rápida de una situación. Esta carga solo se reduciría cuando el sujeto tenga un conocimiento avanzado de los mecanismos que le permita identificar rápidamente el tipo de mecanismo para concentrarse en su característica de movimiento.

Los planteamientos anteriores determinaron el diseño de los ambientes computacionales utilizados en el proyecto, los contenidos y la forma en que los mismos serían presentados. A diferencia de estudios anteriores, donde se comparan imágenes estáticas con respecto a animaciones de sistemas mecánicos, lo que se busca comparar es un material que pueda incluir las imágenes estáticas 
y animaciones prediseñadas, acompañadas por textos explicativos, y como elemento diferenciador un simulador que no se limita a realizar una animación sino que, por el contrario, permita crear y modificar las condiciones de ubicación en el espacio y restricciones de movimiento, brindando la posibilidad de comparar los resultados de dos o más mecanismos similares a los cuales se les cambia uno o varios parámetros, como longitudes y posición de los eslabones que puedan afectar el comportamiento del sistema.

Los materiales desarrollados tienen como objetivo orientar el aprendizaje para identificar los problemas de cinemática, cómo descomponer un objeto complejo en características y después aplicar transformaciones homólogas a estas características, y cómo elegir una estrategia total que reduzca la carga en la memoria de trabajo.

Se busca entonces, seguir un modelo que permita crear una adecuada animación mental que sea representativa de la cinemática real del mecanismo representado; esta estructura estará basada en las etapas planteadas por Hegarty y Narayanan (1998), para la animación mental, y en Norton (1999), para el modelo cinemático. De esta forma se llega a una serie de etapas que pueden equipararse sin necesidad de ser equivalentes exactos. En la tabla 1 se resumen estas características.

\begin{tabular}{ll}
\hline \multicolumn{1}{c}{ Inferencia del movimiento } & \multicolumn{1}{c}{ Comportamiento cinemático } \\
\hline Descomposición del mecanismo. & Modelos matemáticos de movimiento. \\
\hline Identificación de uniones. & Restricciones de movimiento. \\
\hline Conexión entre eslabones. & Restricciones geométricas (longitudes de eslabones). \\
\hline Cadena causal de eventos. & $\begin{array}{l}\text { Movimiento de eslabones (trayectoria, velocidad, } \\
\text { aceleración). }\end{array}$ \\
\hline Modelo mental. & Movimiento del ensamble. \\
\hline
\end{tabular}

Tabla 1. Comparación entre los procesos de inferencia mental de movimiento y el modelo físico cinemático.

\section{Estructura del hipertexto}

La intencionalidad de la estructuración es buscar que el estudiante racionalice el efecto individual de cada componente de forma que le permita inferir el movimiento de todo el conjunto. El análisis de aplicaciones de mecanismos intenta que el individuo sea capaz de identificar estructuras de mecanismos conocidas en cuanto a tipos de movimientos y configuración de eslabones de forma que se maneje un conocimiento previo en movimiento de mecanismos antes de enfrentarse con situaciones problémicas por resolver. El hipertexto fue desarrollado en Asimetrix Toolbook ${ }^{\circledR} 2005$, utiliza animaciones generadas en Cam Studio $^{\circledR}$ a partir de simulaciones hechas en Working Model $^{\circledR}$ y Visual Nastran ${ }^{\circledR}$ e imágenes diseñadas por el grupo de investigación.

Se destacan tres aspectos fundamentales; el primero de ellos está relacionado con la apreciación del movimiento, describiendo las diferentes formas en que un cuerpo puede cambiar de posición en el espacio. El segundo se maneja a partir de la teoría general de 
los mecanismos donde se describen las restricciones cinemáticas establecidas por los diferentes tipos de unión, así como el cálculo simple de los grados de libertad de un mecanismo. Definida la teoría, se encuentran ejemplos descritos de mecanismos comunes que son base de mecanismos más complejos, y por último, existe una sección de videos en el diseño de la interfaz, con aplicaciones complejas de los mecanismos.

El objetivo primordial entonces se centra en identificar adecuadamente cómo pueden generarse casos particulares de movimiento así como reconocer la forma en que puede transmitirse a partir de dispositivos tecnológicos. La figura 8 se muestra el mapa de navegación del hipertexto que da cuenta de todos los temas que se abordan, y la figura 9 presenta la interfaz del hipertexto.

El contenido, el visual permite recrear situaciones contextualizadas al tema referido en la pantalla. Se prefirió el uso de videos que permitieran recrear apropiadamente la noción de movimiento de los diferentes elementos involucrados en una cadena cinemática conformada por el eslabonamiento de elementos desde la entrada hasta la salida del mecanismo.

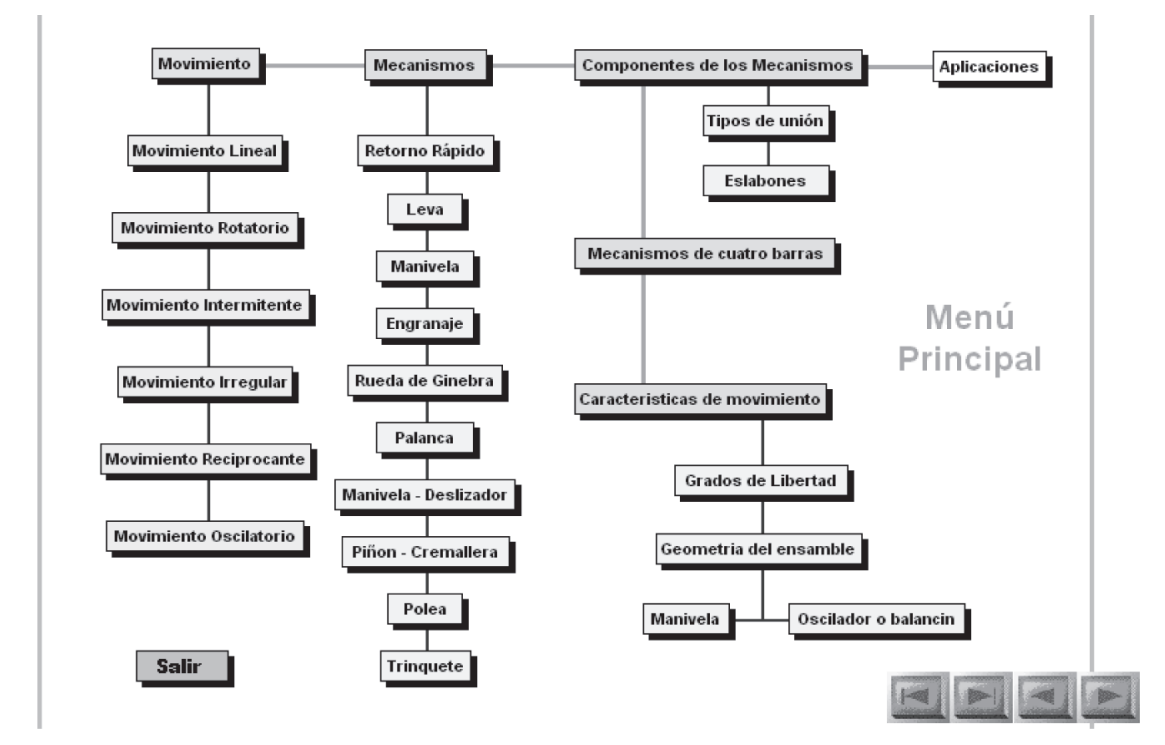

Figura 8. Mapa de navegación del hipertexto. 


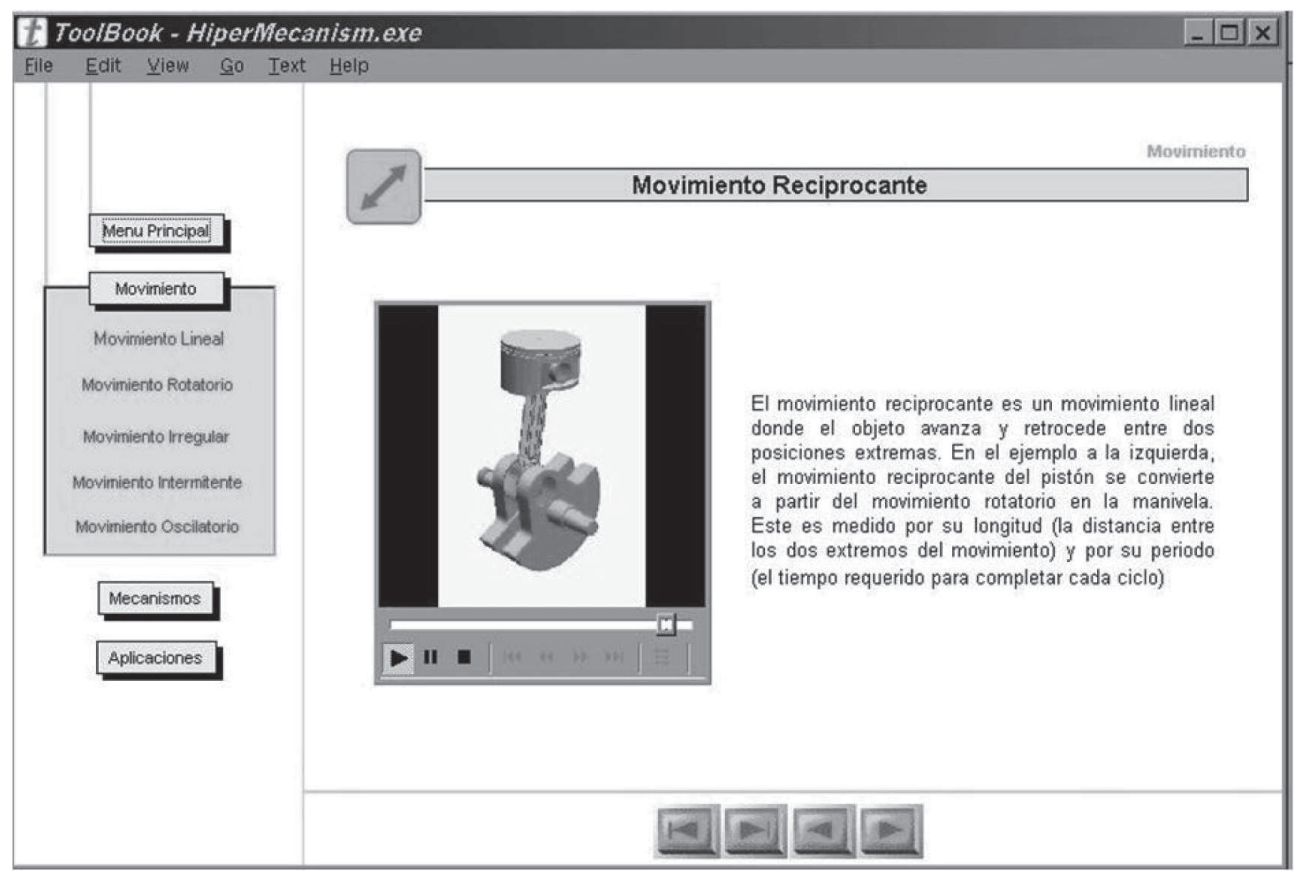

Figura 9. Estructura general de una pantalla en el hipertexto.

\section{Estructura del simulador}

El entorno de trabajo tiene la posibilidad de trabajar formas simples, en un espacio bidimensional, para representar los eslabones, y posee librerías donde se puede escoger y posicionar las uniones entre eslabones de acuerdo con la característica del movimiento, y por último, pueden crearse controles asociados a longitudes y ubicaciones espaciales de los elementos con el fin de modificar rápidamente las características y simular fácilmente el movimiento del conjunto.

Las rutinas de simulación fueron construidas en Working Model ${ }^{\circledR}$ v. 4.0 teniendo en cuenta que es un programa orientado a la simulación cinemática de mecanismos. Siguiendo la estructura que se aprecia en la figura 10, se tiene una zona de trabajo donde se ubican los di- ferentes elementos que intervienen en la simulación. El área mayor corresponde a la representación del mecanismo, a la izquierda se ubicaron los elementos que permiten cambiar las propiedades de ubicación de uniones y de longitudes de eslabones, en la región superior derecha se ubicaron mensajes con instrucciones simples para que el estudiante se contextualizara con la situación presentada en la rutina. En la parte superior izquierda se ubica el título de la rutina.

Según el tema por trabajar, existe un modelo previo para empezar a simular y modificar variables; o en otros casos el estudiante debía construir el mecanismo a partir de diferentes elementos que se le proporcionaron. En total se desarrollaron catorce rutinas de simulación. 


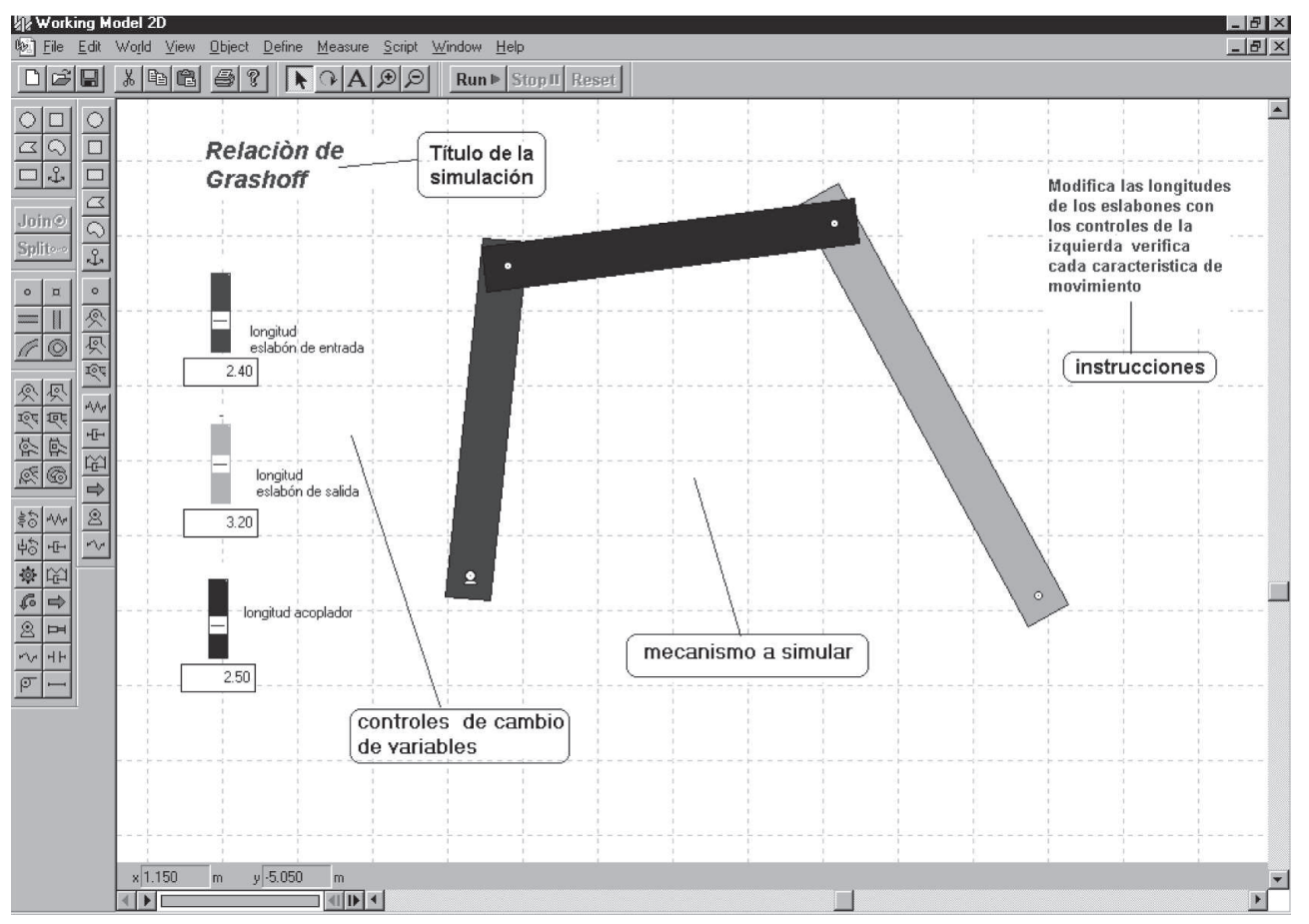

Figura 10. Área de trabajo para la simulación.

\section{Metodología}

La investigación se contextualizó en el marco de la didáctica de la tecnología, en la temática de mecanismos, específicamente en mecanismos planares eslabonados de cuatro barras, y se posibilita a través de la incorporación de un ambiente de aprendizaje computarizado con características particulares de un hipertexto y un simulador enfocado en la solución de problemas en la temática expuesta anteriormente.

\section{Diseño de la investigación}

Se diseñó un ambiente computacional Hipertexto: Mecanismos, el cual presenta los fundamentos conceptuales de la teoría de mecanismos, apoyados con simu- laciones de movimiento de mecanismos y aplicaciones reales. En la tabla 2 se resume el diseño de la investigación.

\begin{tabular}{lcc}
\hline \multicolumn{2}{c}{$\begin{array}{c}\text { Variable } \\
\text { independiente }\end{array}$} & $\begin{array}{c}\text { Variable } \\
\text { dependiente }\end{array}$ \\
\hline $\begin{array}{l}\text { Hipertexto- } \\
\text { simulador }\end{array}$ & Hipertexto & Eficacia \\
\hline Grupo A & Grupo B & Eficacia \\
\hline
\end{tabular}

Tabla 2. Variables involucradas en el diseño experimental.

Las variables independientes corresponden a los grupos experimentales los cuales son determinados de acuerdo con la forma como van a interactuar con la herramienta y las variables dependientes 
corresponden a la eficiencia y la eficacia definidas como:

Eficiencia=Eventos exitosos/ Tiempo empleado (1)

Eficiencia=Eventos exitosos/Total de eventos utilizados (2)

\section{Población, muestra}

y procedimientos experimentales

El escenario experimental se validó con 60 estudiantes de cuarto semestre de Licenciatura en Diseño Tecnológico de la Universidad Pedagógica Nacional. Se distribuyeron aleatoriamente en dos grupos: A y B, de 30 estudiantes cada uno. En el presente estudio el grupo A se caracteriza por interactuar únicamente con el hipertexto. El grupo B se caracteriza por interactuar con el conjunto hipertexto-simulador. La validación se realizó en dos momentos, a saber:

Etapa de interacción. El estudiante está en contacto con las herramientas. El objetivo básico es entender el movimiento de los mecanismos planares de cuatro barras a través del reconocimiento de las diferentes relaciones que existen entre los eslabones, su geometría, sus tipos de unión y el tipo de movimiento de entrada. Así mismo se busca determinar cómo este conjunto de aspectos afectan la característica global de movimiento del mecanismo en el espacio. La interacción con el hipertexto se realizó en tres sesiones de sesenta minutos, y la interacción con el hipertexto-simulador, en tres sesiones de noventa minutos.

Etapa de experimentación. Los estudiantes resuelven cinco problemas en los que se indaga alrededor del comportamiento de los mecanismos y se verifica el proceso que siguieron los estudiantes para llegar a una conclusión. Las sesio- nes de trabajo fueron simultáneas para todos los grupos. El tiempo máximo para el desarrollo de los problemas se estableció de una hora y cincuenta minutos. Se registró el tiempo empleado en la solución de cada problema resuelto y se totalizó el tiempo empleado para resolver los problemas.

La evaluación se hizo planteando cinco mecanismos a los trabajados tanto en el hipertexto como en el simulador y se realizaron preguntas alrededor del movimiento del mecanismo. Las preguntas tenían la intencionalidad de verificar la construcción de la cadena causal de eventos previa a la animación mental. Se hizo un registro personalizado de cada usuario con el fin de recopilar la información de la experimentación.

Teniendo en cuenta que cada individuo puede generar una estrategia diferente para resolver el problema se determinó una cadena causal de eventos estándar la cual tenía los pasos mínimos requeridos para resolver el problema de acuerdo al análogo cinemático propuesto en cada caso y poder establecer cuales eventos de los planteados por el estudiante eran exitosos y así determinar las variables del estudio, eficiencia y eficacia, respectivamente.

\section{Resultados}

Para el análisis de los datos obtenidos se utilizó el análisis de varianza con prueba $F$ para establecer las diferencias entre las medias de los grupos A, y B para la eficiencia y la eficacia en la solución de problemas por parte de los sujetos.

En este estudio se evaluó el efecto de la variable independiente, grupo sobre las variables dependientes, mediante el análisis de varianza (ANOVA) en cada 
problema, se utilizan como variables dependientes: la eficiencia y la eficacia. Esta información se analiza por grupos, de tal forma que la prueba estadística aplicada, muestra si existen diferencias significativas entre ellos.

\section{Comparación de la eficiencia por grupos}

En la figura 11 se aprecia que existe una eficiencia más alta en el grupo que interactúa con el simulador. Es de anotar que el grupo A mantuvo un nivel de eficiencia casi constante; llama la atención debido a que se mantuvo independiente de la dificultad del problema, esto puede suponer que los individuos que trabajaron únicamente con el hipertexto establecen reglas muy similares para la cadena de eventos sin diferenciar las particularidades de cada mecanismo.

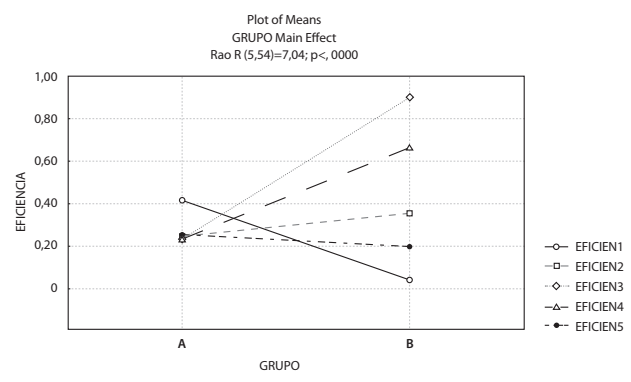

Figura 11. Eficiencia por grupo experimental.

\section{Comparación de la eficacia por grupos}

En la figura 12 se aprecia un aumento significativo en la eficacia del grupo B con respecto al grupo A. Los niveles de eficacia en el primer grupo muestran bajos niveles de eventos exitosos, lo cual muestra que el hipertexto no contribuyó de manera significativa en el aprendizaje de los estudiantes y que evidentemente el uso del hipertexto simulador aportó en el mejoramiento del razonamiento cinemático de los individuos.

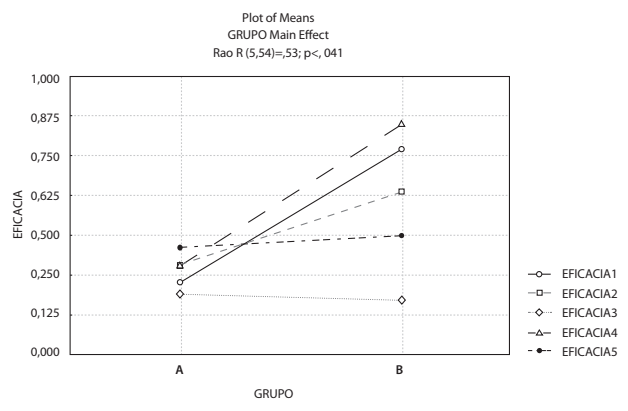

Figura 12. Eficacia por grupo experimental.

\section{Discusión de resultados}

Los resultados obtenidos pueden entenderse, teniendo en cuenta que al utilizar el simulador el individuo está construyendo simultáneamente el modelo mental de movimiento junto con el modelo cinemático, lo cual le permite identificar con mayor claridad las interacciones entre eslabones, uniones y tipos de movimiento generados; sin embargo, este proceso puede aumentar la carga en la memoria de trabajo, por lo que se toma más tiempo para comprender de manera adecuada cada situación. No obstante los resultados se encuentran dentro de lo presupuestado por el grupo frente al simulador que buscaba que le permitiera al estudiante reconocer más fácilmente la interacción entre elementos y le tomara menos tiempo inferir un movimiento.

Además se verificó que el estudiante que tiene la posibilidad de participar en la construcción virtual del mecanismo y en su simulación final es capaz de establecer cadenas causales de eventos más detalladas, logrando inferir el movimien- 
to de una manera más acertada. El grupo que experimentó únicamente con el hipertexto estableció de manera constante un número de eventos exitosos en cada situación; sin embargo, esta condición no es la ideal ya que los requerimientos frente a los elementos que interactuaban en cada problema aumentaban con el problema, por lo que se presentaron respuestas erradas o no esperadas con mucha más frecuencia que en el grupo intervenido con el simulador.

Lo concluido alrededor de la eficacia entra en concordancia con lo analizado para la eficiencia, por cuanto el estudiante del grupo que interactúa con el simulador busca establecer en detalle el modelo que le ayude a inferencias acertadas de movimiento, por tanto, para ello requiere más tiempo debido a que genera y utiliza más información para obtener una respuesta que solucione adecuadamente el problema.

Una nueva pregunta surge entonces con respecto a los resultados: ¿Cuál de los dos indicadores analizados puede llegar a ser más importante en la resolución de problemas de cinemática de mecanismos? Al esperar que el estudiante pueda inferir correctamente el movimiento de cualquier elemento que conforma el mecanismo y, por supuesto, el movimiento de todo el ensamble, la respuesta podría orientarse a que es, posiblemente, más importante el hecho de poder completar un modelo de animación mental detallado y que no dé paso a ambigüedades que puedan producir errores en la solución final, a expensas del tiempo empleado para tal fin.

La eficacia tiene en cuenta la cantidad de eventos exitosos desarrollados por un individuo para resolver un proble- ma, es determinante cuando se trata de analizar situaciones que involucran movimientos complejos, teniendo en cuenta que en este tipo de problemas es importante establecer adecuadamente una secuencia de operaciones tendientes a elaborar un modelo detallado de lo que se observa.

Sin embargo, preocupa que se requiera mucho tiempo para resolver el problema, debido a que el individuo puede agregar más información de la necesaria en los procesos y llevarlo a conclusiones diferentes, como ocurrió en los problemas más complejos.

Una de las condiciones que entra a jugar es la experticia que un individuo pueda desarrollar para resolver problemas de este tipo; esto quiere decir que es capaz de guardar cadenas de eventos parciales y enlazarlas con la nueva situación a la que se ve enfrentado, lo cual llevaría al individuo a resolver los problemas con una buena probabilidad de éxito con un tiempo inferior al que utilizaría una persona que resuelve este tipo de situaciones por primera vez. Queda la inquietud frente a la cantidad y tipo de problemas que debe resolver una persona para generar una condición como la descrita anteriormente; el alcance experimental no logró determinarlo debido a la corta duración de las pruebas, por lo que se propone como un futuro tema de investigación.

La ubicación y percepción espacial es un inconveniente para estudiantes incluso universitarios, la formación alrededor de la concepción espacial teórica no es complementaria con el mundo real, de modo que los modelos matemáticos y geométricos en ocasiones no son fácilmente relacionables con las condicio- 
nes reales, esto hace que el individuo entienda el movimiento desde el punto de vista geométrico y físico en entornos coordenados de forma rectangular, polar e incluso cilíndrica o esférica, pero le es difícil inferir en el espacio real sin sistemas coordenados. Contextualizar desde etapas previas a la formación universitaria, alrededor del movimiento, la transformación y las relaciones causa efecto en cadenas eslabonadas ayudaría significativamente a las personas que se decidan a estudiar modos de movimiento más complejos de forma que su concepción espacial sea más abierta y puedan relacionar más fácilmente todas las variables implícitas en este tipo de situaciones. $\boldsymbol{\Delta}$

\section{Referencias}

Chandrasekaran B. (2000). Multimodal perceptual representations and design problem solving. Invited paper, visual and spatial reasoning in design: Computational and cognitive approaches, MIT, Cambridge, USA.

Hegarty M. (1992). Mental animation: Inferring motion from static displays of mechanical systems. Journal of Experimental Psychology: Learning, Memory and Cognition,18, 1084-1102.

Hegarty, M. and Narayanan H. (1998). Designing Hypermedia Manuals to explain how Machines Work: Lessons from Evaluation of a Theory-based Design Technical Report CSE98-04.

Hegarty, M. and Kozhevnikov, M. (1999). Spatial abilities, working memory and mechanical reasoning. In J. Gero and B. Tversky (Eds.). Visual and Spatial
Reasoning in Design. Key Center for Design and Cognition, Sydney, Australia.

Nikam, D., Sathyarayana, D. and Krishnamurty, S. (2004). Design and Development of Kinematics Visualization Tutor.

Norton, R. (1999). Diseño de maquinaria. 2. ${ }^{\mathrm{a}}$ ed., en español. McGraw Hill.

Poli, C., Fisher, D. L., Pollatsek, A., and Woolf, B.P. (2003). Design for stamping Isolating Pedagogically Effective Components in Multimedia. Tutors and the Classroom. Journal of Engineering Education.

Potter C. and Van der Merwe, E. (2002). Teaching visualization in engineering graphics: Methodology and implications. 